\title{
Preparation and Photocatalytic Performance of Bamboo-Charcoal-Supported Nano-ZnO Composites
}

\author{
Yunlong ZHOU, Zhibiao HU *, Manxia TONG, Qiaoling ZHANG, Changqing TONG \\ College of Chemistry and Materials Science, Longyan University, 364012 No. 1 Dongxiao North Road, Longyan, Fujian \\ Province, China
}

crossref http://dx.doi.org/10. 5755/j01.ms.24.1.17397

Received 09 January 2017; accepted 22 May 2017

\begin{abstract}
Nano-ZnO/bamboo charcoal composites were prepared by precipitation with bamboo charcoal as support. Nano$\mathrm{ZnO} /$ bamboo charcoal composites were characterized by XRD, SEM and EDS. Photocatalytic degradation processes of methyl orange were studied. The results indicate that the structure of nano-ZnO is of the wurtzite type and the grain size is about $19-54 \mathrm{~nm}$. The best preparation temperature for these composites is $500{ }^{\circ} \mathrm{C}$. The composites have better photocatalytic degradation ability than pure $\mathrm{ZnO}$ under UV irradiation. Photocatalytic degradation of methyl orange with the composites obeys first-order kinetics, and the composites can be recycled.

Keywords: Nano-ZnO, charcoal, photocatalytic degradation, methyl orange, precipitation.
\end{abstract}

\section{INTRODUCTION}

Photocatalytic oxidation and degradation of organic pollutants in water, which is part of "green technology" and "environmentally friendly technology", is a new kind of water treatment technology with great potential. It has the advantages of simple process, excellent treatment effects, easily controlled operation conditions and strong oxidation ability. Furthermore, under room temperature and ambient pressure, it can thoroughly decompose organic pollutants of stable structures in water into carbon dioxide, water and other simple inorganic compounds by non-selective oxidation in sufficient reaction time, which results in the avoidance of secondary pollution and offers broad application prospects. In recent years, studies on the photocatalytic degradation of different organic pollutants using semiconductors as catalysts have attracted the attention of scholars from different fields. Especially since photocatalytic oxidation methods using semiconductors were used to deal with organic pollutants in water, they have drawn even more attention and developed so rapidly that they have become a hot topic for water treatment [1].

As a new sort of wide-band gap photocatalytic semiconductor situated in the II-VI group with outstanding performance, zinc oxide $(\mathrm{ZnO})$ has a forbidden band whose width is $3.12 \mathrm{eV}$. Under UV irradiation, $\mathrm{ZnO}$ can produce photo-induced electron-hole pairs, resulting in excellent catalytic performance [2]. Thus, nano-ZnO photocatalytic materials have become a new kind of environmentally friendly materials which have been studied extensively. Since it has the advantage of nontoxicity, low-cost, etc, it is often used in light catalysis [3-5]. While pure nano- $\mathrm{ZnO}$ agglomerates easily, it is difficult to recycle. Bamboo charcoal is an ideal photocatalytic carrier in that it has high porosity and large specific surface area which result in the strong adsorption ability on surface [6-7]. Therefore, in this paper, we took

\footnotetext{
* Corresponding author: Tel.: +86597 2790229.

E-mail address: zhibiaohu@163.com (Z.Hu)
}

advantage of adsorption properties of bamboo charcoal, fixing nano- $\mathrm{ZnO}$ photocatalyst onto the granulated bamboo charcoal to make nano- $\mathrm{ZnO} / \mathrm{bamboo}$ charcoal composites, and combining adsorption with photocatalytic technology which is facilitated to recycle the composite materials. At the same time, we explored the feasibility of nano$\mathrm{ZnO} /$ bamboo charcoal composites for advanced treatment of dye wastewater.

\section{EXPERIMENTS}

\subsection{Instruments and materials}

Instruments: WJF-2000 spectrophotometer (UNICO Instrument Co. Ltd, China), digital $\mathrm{pH}$ meter (Albert Instrument Factory, China), electronic balance (Sartorius, Germany, BS323S), 8411A electric vibration sieve machine (Xiangtan City Kelian Analysis Instrument Co. Ltd, China), THD-1015 low-temperature thermostat bath (Ningbo Tianheng Instrument Factory, China), 300W UV lamp (Shanghai Yaming Lighting Co. Ltd, China), a SKG05123K vacuum oven ( Zhonghuan Experimental Oven Co. Ltd, China), Quanta650 scanning electron microscope (FEI Company, USA), DX2700 X-ray diffractometer (Dandong Fangyuan Instrument Co. Ltd, China).

Materials: bamboo charcoal (Shengda Technology Co. Ltd, China); ammonium carbonate, zinc nitrate, nitric acid, methyl orange (these reagents are analytically pure, from Guangdong Xilong Chemical Factory, China).

\subsection{Preparation of nano- $\mathrm{ZnO} / \mathrm{bamboo}$ charcoal composites and nano- $\mathrm{ZnO}$}

Pre-treatment of bamboo charcoal: Using the 8411A electric vibration sieve machine, the $0.082 \mathrm{~mm}-0.164 \mathrm{~mm}$ bamboo charcoal particles (with BET surface area of $930 \mathrm{~m}^{2} / \mathrm{g}$ ) were washed successively by tap water and distilled water after steeping in diluted acid for 24 hours, then dried at $120{ }^{\circ} \mathrm{C}$.

Preparation of composites by precipitation method: $50 \mathrm{ml}$ of the $0.5 \mathrm{~mol} / \mathrm{L}\left(\mathrm{NH}_{4}\right)_{2} \mathrm{CO}_{3}$ and $0.5 \mathrm{~mol} / \mathrm{L} \mathrm{Zn}\left(\mathrm{NO}_{3}\right)_{2}$ 
solutions were prepared and mixed in the same volume ratio after sufficient reaction by stirring for 5 minutes in a magnetic stirrer. $4.00 \mathrm{~g}$ of bamboo charcoals were added into the mixture in the process of stirring. After several minutes, extraction filtration was carried out as the target bamboo charcoals were loaded by the mixture uniformly. Dried at $110{ }^{\circ} \mathrm{C}$, those charcoals were then switched into a crucible and sintered respectively in vacuum at $300{ }^{\circ} \mathrm{C}$, $400{ }^{\circ} \mathrm{C}, \quad 500{ }^{\circ} \mathrm{C}, \quad 600{ }^{\circ} \mathrm{C}$ and $700{ }^{\circ} \mathrm{C}$. Taken out after cooling and washed by distilled water, they were filtered and dried again, with the composite materials of nano$\mathrm{ZnO} /$ bamboo charcoal as the final product.

For a comparative purpose, the pure $\mathrm{ZnO}$ was prepared by the same procedure and sintered in vacuum at $500{ }^{\circ} \mathrm{C}$ without the addition of bamboo charcoals.

\subsection{Characterization of composites}

The phase characterization was carried out by the Xray diffractometer (PANalytical,Holland) with the conditions as following: $\mathrm{Cu}$ anode, $\mathrm{K} \alpha=1.5405 \times 10^{-10} \mathrm{~m}$, $30 \mathrm{kV}$ voltage, $25 \mathrm{~mA}$ current, scanning step $0.030^{\circ}$ and scanning range $2 \theta=20-50^{\circ}$.

The morphology was observed by the Quanta650 scanning electron microscope (HITACHI,Japan), while the chemical composition of micro-areas was detected by energy dispersive spectrometer (EDS).

\subsection{Test on photocatalytic degradation of methyl orange with the composites}

A certain amount of composites was put into a $100 \mathrm{~mL}$ beaker in which a certain concentration of methyl orange was added. Irradiated by UV light source at constraint temperature, the composites were filtered after standing for 30 minutes. And then the absorbance of residual methyl orange was determined at the wavelength of $464 \mathrm{~nm}[8,9]$. The degree of degradation of methyl orange was calculated by the following equation according to the variation of concentration before and after the light exposure $[10,11]$ :

$D \%=\left(A_{0}-A\right) / A_{0} \times 100 \%$,

where in $D$ is the degree of degradation, $A_{0}$ and $A$ are the dye absorbance at time 0 and t respectively.

\section{RESULTS AND DISCUSSION}

\subsection{XRD analysis}

Fig. 1 is the XRD pattern of the composites calcined at different temperatures in the range $300-700{ }^{\circ} \mathrm{C}$. The locations of all diffraction peaks (100), (002), (101), (102) correspond to hexagonal $\mathrm{ZnO}$ (wurtzite). Bamboo charcoal did not form diffraction peaks in the XRD pattern, confirming that because it is a kind of amorphous carbon [12]. Scherrer equation was used to define the crystallite size:

$D=\left(\frac{K \lambda}{B}\right) \cdot \cos \theta$,

where $D$ is the crystallite size, $K$ is the Scherrer constant, $B$ the half width of diffraction peak, $\theta$ the diffraction angle, $\lambda$ the wavelength of $X$ ray. Calculated by the equation, the crystallite size was in the range of $19-54 \mathrm{~nm}$, which was close to the particle size of nano $\mathrm{ZnO}$ in the $\mathrm{ZnO} / \mathrm{BC}$ composite prepared by Yu.X.L. indicating that the composites prepared with the above method were nanosize $\mathrm{ZnO}$ [13]. From Fig. 1 it is evident, that the peaks of nano- $\mathrm{ZnO}$ became increasingly sharper as the calcining temperature rises from 300 to $700{ }^{\circ} \mathrm{C}$, indicating an increase of the crystallite size.

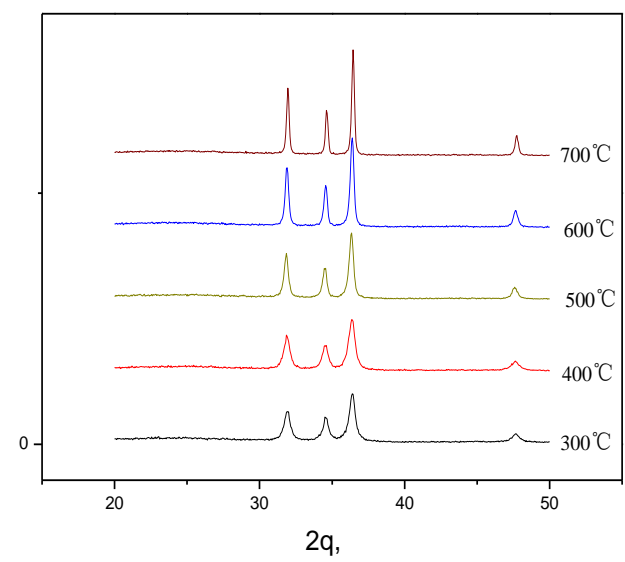

Fig. 1. XRD patterns of the composites calcined at different temperatures in the range $300-700{ }^{\circ} \mathrm{C}$

\subsection{SEM analysis}

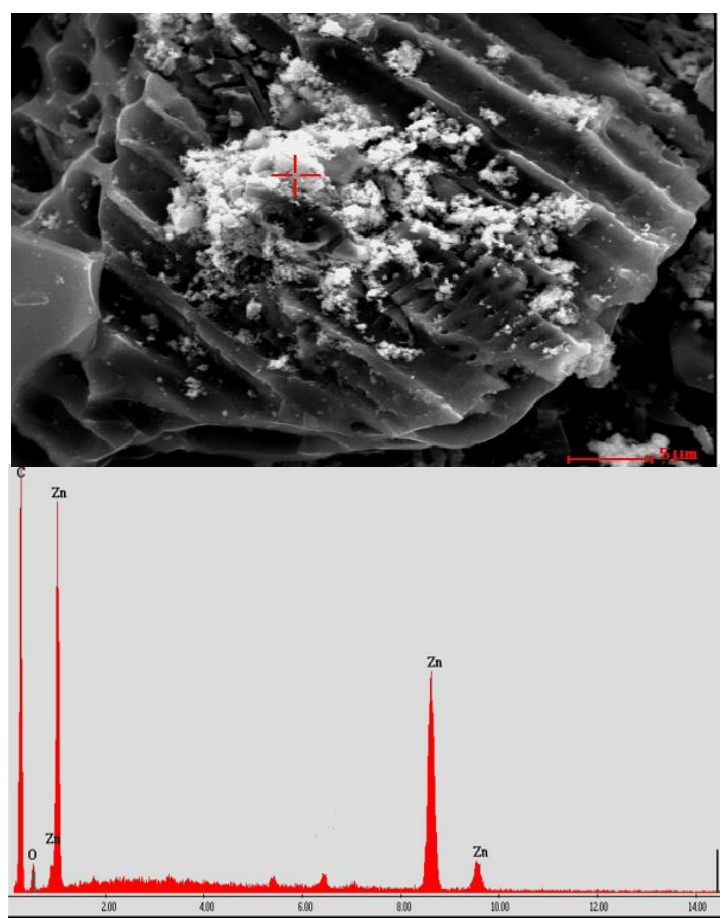

Fig. 2. Nano-ZnO/bamboo charcoal composites calcined at $500{ }^{\circ} \mathrm{C}$ : $\mathrm{a}-\mathrm{SEM}$ image; $\mathrm{b}-$ EDS spectrum

Fig. 2 shows the SEM image and EDS spectrum analysis of nano-ZnO/bamboo charcoal composites. As shown in Fig. 2 a, there were some tiny $\mathrm{ZnO}$ particles on the surfaces of layers of structural bamboo charcoal. In Fig. 2 b, the EDS spectrum of samples, we can see there are three elements ( $\mathrm{Zn}, \mathrm{O}$ and $\mathrm{C}$ ), in which $\mathrm{C}$ is the supporting bamboo charcoal. In conclusion, we have prepared nano-size $\mathrm{ZnO} /$ bamboo charcoal composites by 
the precipitation method and retained charcoal even after calcination to $500{ }^{\circ} \mathrm{C}$.

\subsection{Influence of calcining temperature on photocatalytic properties}

$0.500 \mathrm{~g}$ of nano-ZnO/bamboo charcoal composites prepared at different temperatures were weighed in, and added into $50.0 \mathrm{~mL}$ methyl orange solution of concentration $3 \times 10^{-5} \mathrm{~mol} / \mathrm{L}$. The concentration of residual methyl orange solution was determined after standing for 30 minutes at room temperature under UV irritation. Then the degree of degradation was calculated. Results are shown in Fig. 3.

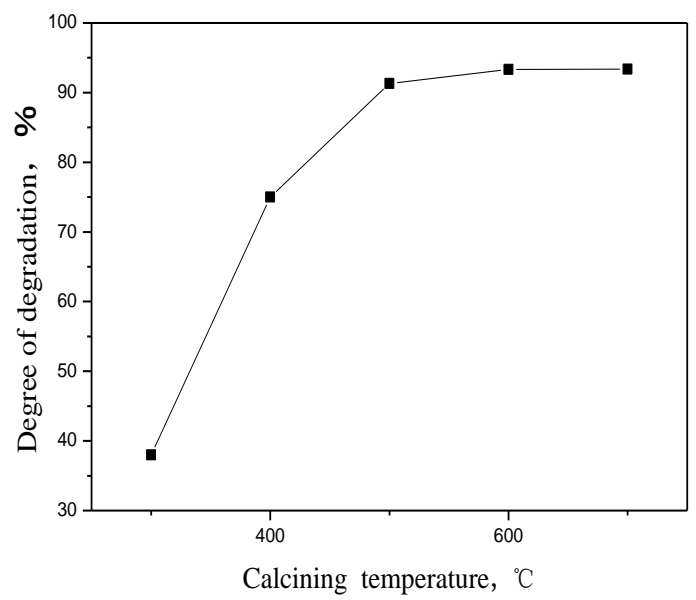

Fig. 3. Photocatalytic performance of composites calcined at different temperatures

As shown in Fig. 3, the photocatalytic performance of the prepared composites on methyl orange gradually improved, when the calcining temperature was increased from 300 to $500{ }^{\circ} \mathrm{C}$ and reached almost its final value at $500{ }^{\circ} \mathrm{C}$.

\subsection{Comparison on photocatalytic degradation performance of different materials}

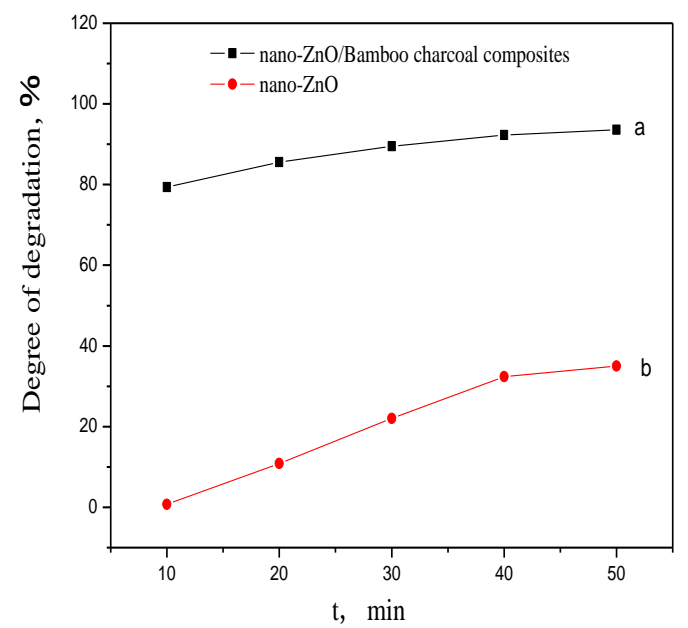

Fig. 4. Kinetics of the degree of degradation of methyl orange: $\mathrm{a}-\mathrm{ZnOn} / \mathrm{bamboo}$ charcoal composites; $\mathrm{b}$-pure nano$\mathrm{ZnO}$

After standing for 40 minutes at room temperature under UV irradiation, the absorption of these samples was determined every 10 minutes. Thus the residual concentration of the methyl orange solution and the degree of degradation was calculated. Results are shown in Fig. 4.

In Fig. 4, we can see that the degree of degradation of pure nano- $\mathrm{ZnO}$ and nano- $\mathrm{ZnO} /$ bamboo charcoal composites was $32.4 \%$ and $92.3 \%$, respectively, after 40 minutes UV irradiation, which indicated that the degree of degradation of is higher when using the composites, and their degradation performance is superior to that of pure nano-ZnO. The porous structure of bamboo charcoal combined with nano- $\mathrm{ZnO}$ evidently increased the contact area between $\mathrm{ZnO}$ composites and methyl orange molecules, leading to the obviously higher degree of degradation of the composites compared to the pure nano$\mathrm{ZnO}$. Yu. X.L. used the composites to degrade rhodamine $\mathrm{B}$ and acid fuchsin and achieved the degradation rate of $93 \%$ and $99 \%$ respectively,showing that nano$\mathrm{ZnO}$ /bamboo composites can degrade different organic pollutants [13].

\subsection{Photocatalytic kinetics}

The curve of $-\ln \left(c / c_{0}\right)$ and time at the room temperature was drawn up. As shown in Fig. 5, we can figure out good linear relationships, in which both of the correlation coefficient $\mathrm{R}$ are greater than 0.98 . The results showed that the photocatalytic degradation of methyl orange on the composite materials and $\mathrm{ZnO}$ is in accordance with the first-order kinetic equation [14]:

$-\ln \left(c / c_{0}\right)=k t$

where $k$ is rate constant, $c_{0}$ and $c$ are the initial concentration and the concentration after degradation of methyl orange respectively, $t$ is the degradation time.

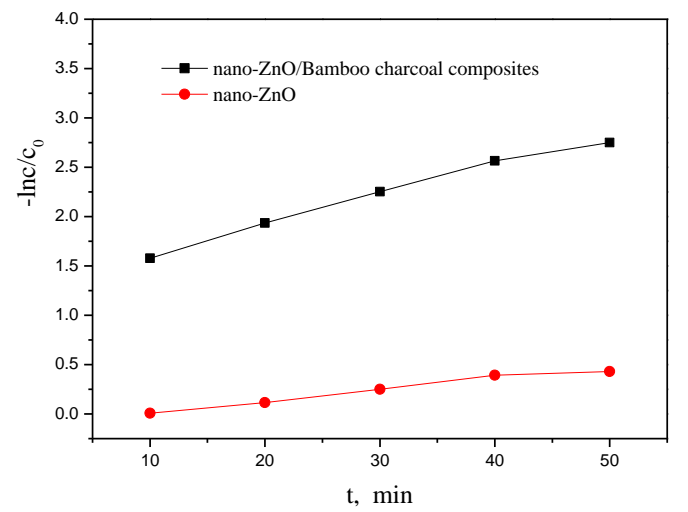

Fig.5. Relationship of $-\ln \left(\mathrm{c}_{\mathrm{c}}\right)$ and time: $\mathrm{a}-\mathrm{ZnOn} / \mathrm{bamboo}$ charcoal composites; $\mathrm{b}$ - nano- $\mathrm{ZnO}$

\subsection{Recycling of composites}

The composites $(0.500 \mathrm{~g})$ were recycled 5 times from methyl orange solution $\left(50 \mathrm{~mL}, 2 \times 10^{-5} \mathrm{~mol} / \mathrm{L}\right)$. composites after each usage were treated by filtering, washed by water and dried at $110^{\circ} \mathrm{C}$ standing for 40 minutes at room temperature under the conditions of UV irradiation. The residual concentration of methyl orange and the degree of degradation after each recycling was determined. Results are shown in Fig. 6.

With reference to Fig. 6 we can see that the photocatalytic degree of degradation of composite materials on methyl orange decreased after repeated 
recycling. This result might be due to the contamination of composites caused by the incomplete removal of intermediate products remaining on the surface of the composites after each washing. Moreover, in the process of reuse, nanometer $\mathrm{ZnO}$ loaded on the surface of carriers might get lost resulting in a decreasing degree of degradation. However, the photocatalytic degree of degradation of composites on methyl orange still retains $64.6 \%$ after recycling for 5 times. In conclusion, the composite materials can be recycled and used repeatedly while still retaining some photocatalytic activity, Li,S.Q., [15] utilized $\mathrm{ZnO} /$ rectorite nanocomposite to degrade methylene blue solution under simulated sunlight. The decolorization rate was more than $80 \%$ after recycling for 5 times, which solves the problems of small size and difficult recovery of nanometer materials.

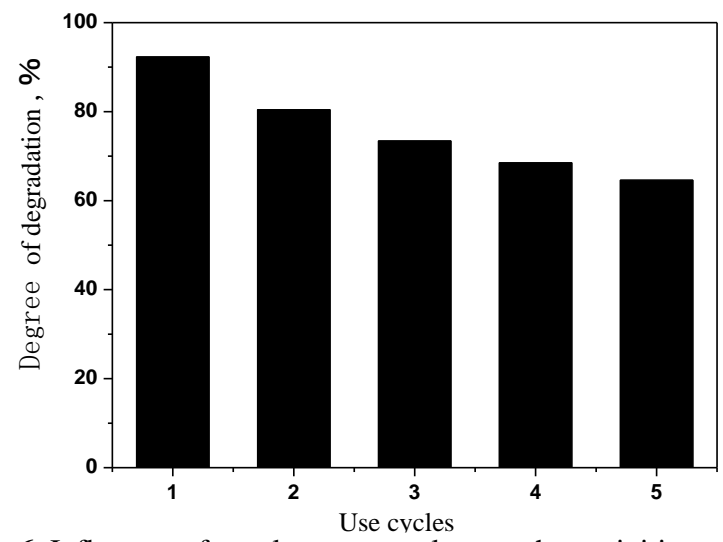

Fig. 6. Influence of catalyst use cycles on the activities of the catalyst composites

\section{CONCLUSIONS}

Nano-ZnO/bamboo charcoal composite materials with hexagonal (wurtzite-type) nano-ZnO, prepared by precipitation have better photocatalytic activities than pure $\mathrm{ZnO}$ under UV irradiation. The photocatalytic degradation performance of these composites is best after calcination at $500{ }^{\circ} \mathrm{C}$. The degradation of methyl orange on nano$\mathrm{ZnO} /$ bamboo charcoal composites is in line with first-order kinetics, and the composites can be readily recycled.

\section{Acknowledgments}

This work was financially supported by" $A$ " project of Provincial Education Department of Fujian(JA12314), "B" project of Provincial Education Department of Fujian(JB12222), the School Research Program of Longyan University (LC2013008) and the Scientific Start Foundation of Longyan University (LB2014001).

\section{REFERENCES}

1. Hoffmann, M.R., Martin, S.T., Choi, W., Bahnemann, D.W. Environmental Applications of Semiconductor Photocatalysis Chemical Reviews 95 (1) 1995: pp. 69-96. https://doi.org/10.1021/cr00033a004

2. Xie, J., Li, Y.T., Zhao, W., Bian, L., Wei, Y. Simple Fabrication and Photocatalytic Activity of $\mathrm{ZnO}$ Particles with Different Morphologies Powder Technology 207 (1-3) 2011:pp. 140-144. https://doi.org/10.1016/j.powtec.2010.10.019
3. Nagaraja, R.,Kottam, N.,Girija, C.R.,Nagabhushanab, B.M. Photocatalytic Degradation of Rhodamine B Dye Under UV/solar Light Using ZnO Nanopowder Synthesized by Solution Combustion Route Powder Technology 215-216 2012: pp. $91-97$.

https://doi.org/10.1016/j.powtec.2011.09.014

4. Wang, L.N., Zheng, Y.Y., Li, X.Y., Dong, W.J., Tang, W.H., Chen, B.Y., Li, C.R., $\quad$ Li, X., $\quad$ Zhang, T.R., Xu, W. Nanostructured Porous ZnO Film with Enhanced Photocatalytic Activity Thin Solid Films 519 (16) 2011: pp. 5673-5678. https://doi.org/10.1016/j.tsf.2011.02.070

5. Fatimah, I.,Wang, S.B.,Wulandari, D. $\mathrm{ZnO} /$ montmorillonite for Photocatalytic and Photochemical Degradation of Methylene Blue Applied Clay Science 53 (4)

2011: pp. 553-560. https://doi.org/10.1016/j.clay.2011.05.001

6. Wu, C.H., Shr, J.F., Wu, C.F., Hsiec, C.T. Synthesis and Photocatalytic Characterization of Titania-supported Bamboo Charcoals by Using Sol-gel Method Journal of Materials Processing Technology 203 (1-3) 2008: pp. 326-332. https://doi.org/10.1016/j.jmatprotec.2007.10.073

7. Chuang, C.S., Wang, M., Ko, C.H., Ou, C.C., Wu, C.H. Removal of Benzene and Toluene by Carbonized Bamboo Materials Modified with $\mathrm{TiO}_{2}$ Bioresource Technology 99 (5) 2008: pp. 954-958. https://doi.org/10.1016/j.biortech.2007.03.003

8. Liu, Y.C., Zhang, G.M., Chong, S., Zhang, N., Chang, H.Z., Huang, T., Fang, S.Y. $\mathrm{NiFe}\left(\mathrm{C}_{2} \mathrm{O}_{4}\right)_{\mathrm{x}}$ as a Heterogeneous Fenton Catalyst for Removal of Methyl Orange Journal of Environmental Management 192 2017: pp. 150-155.

https://doi.org/10.1016/j.jenvman.2017.01.064

9. Wu, X., Zhang, D., Jiao, F.P., Wang, S. Visible-light-driven Photodegradation of Methyl Orange Using $\mathrm{Cu}_{2} \mathrm{O} / \mathrm{ZnAl}$ Calcined Layered Double Hydroxides as Photocatalysts Colloids and Surfaces A: Physicochemical and Engineering Aspects 508 2016: pp. $110-116$. https://doi.org/10.1016/j.colsurfa.2016.08.047

10. Fu, M., Li, Y.l., Wu, S.W., Lu, P., Liu, J., Dong, F. Sol-gel Preparation and Enhanced Photocatalytic Performance of $\mathrm{Cu}-$ doped ZnO Nanoparticles Applied Surface Science 258 (4) 2011: pp. $1587-1591$. https://doi.org/10.1016/j.apsusc.2011.10.003

11. Akyol, A., Yatmaz, H.C., Bayramoglul, M. Photocatalytic Decolorization of Remazol Red RR in Aqueous $\mathrm{ZnO}$ Suspensions Applied Catalysis B: Environmental 54 (1) 2004: pp. $19-24$ https://doi.org/10.1016/j.apcatb.2004.05.021

12. Wang, X.J., Wang,Y., Wang, X., Liu, M., Xia, S.Q., Yin, D.Q., Zhang, Y.L., Zhao, J.F. Microwave-assisted Preparation of Bamboo Char coal-based Iron-containing Adsorbents for $\mathrm{Cr}(\mathrm{VI})$ Removal Chemical Engineering Journal 174 (1) 2011:pp. 326-332. https://doi.org/10.1016/j.cej.2011.09.044

13. Yu, X.L., Qin, A.M., Liao, L., DU, R., Tian, N., Huang, S., Wei, Ch. Removal of Organic Dyes by Nanostructure ZnOBamboo Charcoal Composites with Photocatalysis Function Advances in Materials Science and Engineering 2015 2015: pp. 1-6. http://dx.doi.org/10.1155/2015/252951

14. Sobana, N., Muruganandam, M., Swaminathan, M. Characterization of AC-ZnO Catalyst and Its Photocatalytic Activity on 4-acetylphenol Degradation Catalysis Communications 9 (2) 2008: pp. 262-268. https://doi.org/10.1016/j.catcom.2007.04.040

15. Li, S.Q., Zhou, P.J., Zhang, W.S., Chen, S., Peng, H. Effective Photocatalytic Decolorization of Methylene Blue Utilizing $\mathrm{ZnO} /$ rectorite Nanocomposite Under Simulated Solar Irradiation Journal of Alloys and Compounds 616 2014: pp. $227-234$

https://doi.org/10.1016/j.jallcom.2014.07.102 